\title{
A Combination of STATCOM and Distribution Transformer With Passivity-based Control for Reactive Power Compensation
}

\author{
Jinmu Lai ${ }^{1, a}$, Xianggen Yin ${ }^{1, b}$, Ertao Lei ${ }^{1, c}$ and Yu Chen ${ }^{1, d}$ \\ ${ }^{1}$ State Key Laboratory of Advanced Electromagnetic Engineering and Technology, Huazhong \\ University of Science and Technology, Wuhan 430074, China. \\ aaijinmu@126.com, ${ }^{b} x g y i n @$ hust.edu.cn, ${ }^{\mathrm{a}}$ leiertao2008@163.com, ${ }^{\mathrm{d}} 744705273 @ q q . c o m$
}

\begin{abstract}
Keywords: Distribution Transformer, STATCOM, Reactive Power Compensation, Passivity-Based control, Injection Damping.

Abstract. This paper studies static synchronous compensator (STATCOM) accessed in the system via intermediate taps on transformer high voltage wingdings. For Dyn11 type transformers, the proposed circuit configuration of DT-STATCOM is analyzed, and the function of reactive power compensation is verified. Then, the Euler-Lagrangian (EL) model of a distribution transformer-static synchronous compensator (DT-STATCOM) is established. This paper also proposes an improved $i_{p}-i_{q}$ current detection method. Meanwhile, the passivity-based control (PBC) law which can decouple active and reactive power is proved. By selecting proper injecting damping, we can optimize the dynamic performance and realize fast compensation of reactive power. Finally, simulation results show the effectiveness of the proposed structure with $\mathrm{PBC}$ in reactive power compensation.
\end{abstract}

\section{Introduction}

Static synchronous compensator (STATCOM) is an important device for reactive power compensation. Currently, there are two trends for STATCOM technology. One is the development of high voltage transmission-type STATCOM (T-STATCOM) [1,2] with the goal of high-voltage transmission system reactive power compensation and voltage support; the other is distributed-STATCOM (D-STATCOM) $[3,4]$ to improve the power quality of the consumer side. However, both methods have advantages and disadvantages. Besides, distribution transformer is widely used in distribution network, and its reactive power consumption can't be ignored at low load factor (less than 0.3) [5]. This paper proposes a novel integrated structure of STATCOM and distribution transformer. STATCOM is connected to the winding taps on the primary windings for compensation current injection. The structure is helpful to make full use of the spare capacity of the transformer and increase the capacity utilization. The reactive power cost of the transformer itself can also be compensated. At the same time, a suitable windings taps can be selected to reduce the voltage stress, which is helpful to decrease the cascade count of H-bridge cells.

It is important to design control algorithm of STATCOM which directly affects the performance of reactive power compensation. Currently, the research of control algorithm is conducted in-depth by domestic and foreign scholars. Although linear control strategies have been widely used in the STATCOM system and have obtained the certain achievements, it is not robust to the parameter variations which may adversely affect the performance. In fact, STATCOM is a non-linear, strong coupling, multivariable system. Nonlinear control has already been used in STATCOM system. Despite their satisfactory performance, nonlinear control methods suffer from the complexity in implementation which is difficult to apply in the engineering projects. The aforementioned control methods show various advantages and disadvantages related to stability, dynamic response, steady state error, robustness against parameter variations, switching frequency and control complexity. However, the dissipation properties of STATCOM which are inherently existed in its physical structure are not taken into consideration by aforementioned methods. Passivity is a restatement of energy conservation in STATCOM system, and plays an important role in designing controller to attain global asymptotic stability and command current tracking. Passivity-based control method (PBC) is an effective tool for nonlinear system, and was first introduced into power electronics 
control by Ortega. R. [6]. PBC method reshapes energy storage and injects damping [7-9]. It can make STATCOM system converge to the desired stable equilibrium points and accelerate energy dissipation.

This paper proposes a novel reactive current calculating method which applies to DT-STATCOM based on the instantaneous reactive power theory. Furthermore, the mathematical model of DT-STATCOM system based on E-L function is established and a PBC method which is verified by Lyapunov stability is proposed for DT-STATCOM. Finally, the simulation tests based on Matlab/Simulink show that the proposed structure of DT-STATCOM and control method which applies to system have a good compensation performance.

\section{Structure of DT-STATCOM}

Currently, the distribution transformers are mainly categorized into Dyn-type, Yyn-type and Yzn-type by winding connection. In order to improve the endurance ability of unbalanced load and harmonic, Dyn11 type distribution transformer is widely used in distribution network. Hence, this paper primarily discusses Dyn11-type distribution transformer integrated with STATCOM.

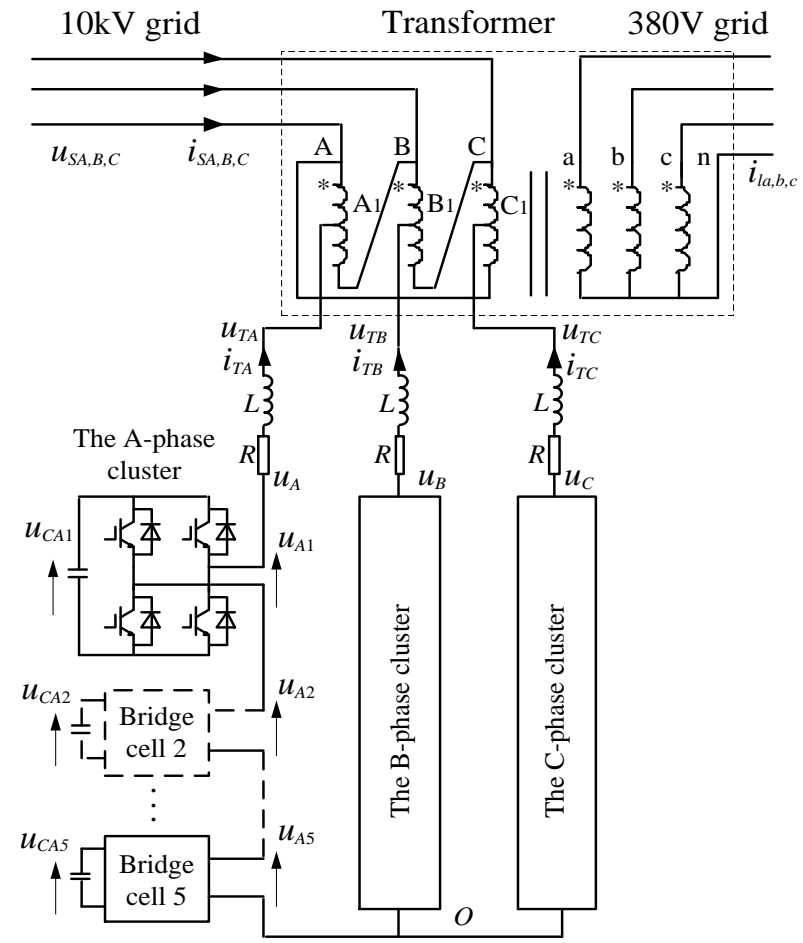

Fig.1. Circuit configuration of the proposed DT-STATCOM.

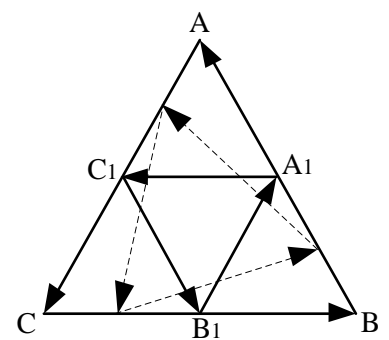

Fig.2 Phasor diagram of line voltage at HV side

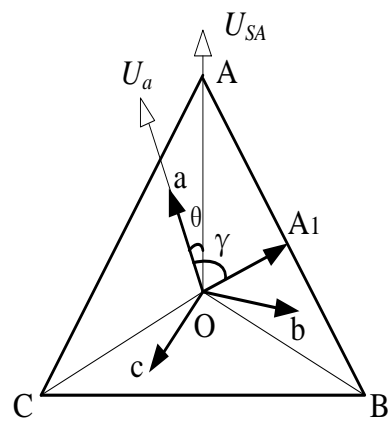

Fig.3. Phasor diagram of Dyn11 transformer.

Notice that, $u_{S A}, u_{S B}$, and $u_{S C}$ are three phase voltage of grid system. $i_{S A}, i_{S B}$, and $i_{S C}$ are three phase current of grid system. $u_{T A}, u_{T B}$, and $u_{T C}$ are three phase voltages at connected taps of HV side of transformer. $i_{T A}, i_{T B}$, and $i_{T C}$ are three phase injected current of STATCOM at connected taps. $u_{a}, u_{b}$, and $u_{c}$ are three phase voltage at low side of transformer. $i_{l a}, i_{l b}$, and $i_{l c}$ are three phase current of load. $L$ is the filter inductor. $R$ is the equivalent resistance of filter inductor. $C$ is the dc capacitor.

Fig. 1 shows the proposed circuit configuration of DT-STATCOM which includes a three-phase CPS-SPWM reactive power compensation device, filter and Dyn-type distribution transformers with intermediate taps. STATCOM can be connected to three taps (shown as $\mathrm{A}_{1}, \mathrm{~B}_{1}, \mathrm{C}_{1}$ in Fig.1) on high-voltage $(\mathrm{HV})$ windings of the transformer. Due to lack of neutral point at the primary side of Dyn transformer, the setting of the taps at HV side should guarantee that line voltages of STATCOM are symmetrical, as shown in Fig. 2. In this structure, we can select a suitable connected voltage level which can reduce costs and simplify the power circuit of STATCOM, but the minimum of connected line voltage is half of the line voltage. Although STATCOM is located at HV side of transformer which is different from load point, only if the compensation current of STATCOM matches the 
system needed, the purpose of dynamic reactive power compensation which includes reactive power of loads and that of transformer consumed can be achieved.

\section{Control Method Design Based on PBC}

\section{A. Improved Reactive Current Detection Method}

For the DT-STATCOM, the compensation currents are injected via the winding taps on the primary windings. The current injected point and load point are not the same point. There are amplitude and phase angle deviation between the load current and compensation current. Therefore, the traditional current detection method can't be used directly.

According to the characteristics of the Dyn11-type transformer, the supply voltage $u_{S A}$ is lagging behind the load voltage $u_{a}$ by $30^{\circ}$, as shown in Fig. 3 . When the central taps are chosen as the connection points, the central taps voltage is lagging behind the load voltage $u_{a}$ by $\gamma\left(\gamma=90^{\circ}\right)$. Meanwhile, the amplitude deviation caused by transformer ratio must be compensated. The load currents must be converted to the injected taps via

$$
i_{\text {Tqref }}=-\frac{i_{q L}}{K}
$$

where $K$ is the transformer voltage ratio.

According to the analysis above, the schematic diagram of improved reactive current detection method is shown as Fig. 4.

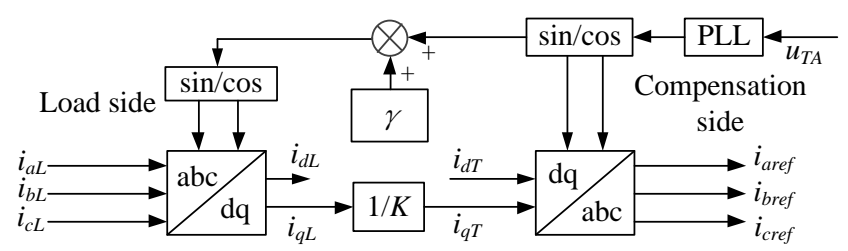

Fig. 4. Schematic diagram of improved $i_{p}-i_{q}$ current detection method.

\section{B. System Modeling}

The mathmatical model of the DT-STATCOM in ABC coordinate is

$$
\left\{\begin{array}{l}
L \frac{d i_{T A}}{d t}=u_{A}-i_{T A} R-u_{T A} \\
L \frac{d i_{T B}}{d t}=u_{B}-i_{T B} R-u_{T B} \\
L \frac{d i_{T C}}{d t}=u_{C}-i_{T C} R-u_{T C}
\end{array}\right.
$$

where

$$
u_{k}=\sum_{n=1}^{5} u_{k n}, k=A, B, C
$$

Applying the $d \_q$ transformation matrix to (2), we can obtain

$$
\left\{\begin{array}{l}
L \frac{d i_{T d}}{d t}=u_{d}+\omega L i_{T q}-i_{T d} R-u_{T d} \\
L \frac{d i_{T q}}{d t}=u_{q}-\omega L i_{T d}-i_{T q} R-u_{T q}
\end{array}\right.
$$

where $i_{T d}$ and $i_{T q}$ are the d-axis and q-axis components of compensation current. $u_{d}$ and $u_{q}$ are the $\mathrm{d}$-axis and q-axis components of STATCOM cluster voltage. $u_{T d}$ and $u_{T q}$ are the d-axis and q-axis components of three phase voltages at connected taps of HV side of transformer. $\omega$ represents the power grid angular frequency. 
The E-L system could describe the characteristics of the system which is hard to be expressed by linearization control method. Equation (4) can be expressed as E-L system model, and we will obtain (5) by selecting $\mathrm{i}_{T d}$ and $i_{T q}$ as state variables.

$$
\boldsymbol{M} \dot{\boldsymbol{x}}+\boldsymbol{J} \boldsymbol{x}+\boldsymbol{R} \boldsymbol{x}=\boldsymbol{F}
$$

where

$$
\begin{aligned}
& \boldsymbol{M}=\boldsymbol{M}^{\mathrm{T}}, \boldsymbol{R}=\boldsymbol{R}^{\mathrm{T}}, \boldsymbol{J}+\boldsymbol{J}^{\mathrm{T}}=\mathbf{0} \\
& \boldsymbol{M}=\left[\begin{array}{ll}
L & 0 \\
0 & L
\end{array}\right], \quad \boldsymbol{J}=\left[\begin{array}{cc}
0 & -\omega L \\
\omega L & 0
\end{array}\right], \quad \boldsymbol{R}=\left[\begin{array}{ll}
R & 0 \\
0 & R
\end{array}\right], \quad \boldsymbol{F}=\left[\begin{array}{l}
u_{d}-u_{T d} \\
u_{q}-u_{T q}
\end{array}\right], \quad \boldsymbol{x}=\left[\begin{array}{l}
i_{T d} \\
i_{T q}
\end{array}\right]
\end{aligned}
$$

The passivity system indicates that the accumulated energy of the system is always less than the sum of energy injected from the outside. PBC method based on E-L model involves two important parts which are energy reshaping and damping injection to accelerate dissipation for system. Energy reshaping part is to maintain the energy distribution at a desired equilibrium point. The damping injection part can accelerate energy dissipation of inverter system and assure that the new working point is globally asymptotically stable.

The storage energy function of STATCOM can be described as

$$
H(x(T))=\frac{1}{2} \boldsymbol{x}^{\mathrm{T}} \boldsymbol{M} \boldsymbol{x}=\frac{1}{2}\left(L i_{T d}^{2}+L i_{T q}^{2}\right)
$$

By taking the derivative of $H(x(T))$ and substituting (5) into (6)

$$
\dot{H}(x(\mathrm{~T}))=L i_{T d} \dot{i}_{T d}+L i_{T q} \dot{i}_{T q}=\boldsymbol{x}^{\mathrm{T}}(\boldsymbol{F}-\boldsymbol{J} \boldsymbol{x}-\boldsymbol{R} \boldsymbol{x})=\boldsymbol{x}^{\mathrm{T}} \boldsymbol{F}-\boldsymbol{x}^{\mathrm{T}} \boldsymbol{R} \boldsymbol{x}
$$

Then (7) can be rewritten in integral form (8).

$$
H(x(\mathrm{~T}))-H(x(0))=\int_{0}^{T} \boldsymbol{x}^{\mathrm{T}} \boldsymbol{F} d \tau-\int_{0}^{T} \boldsymbol{x}^{\mathrm{T}} \boldsymbol{R} \boldsymbol{x} d \tau
$$

where $T$ means the initial time to the current time.

Equation (8) indicates that the accumulation energy [ $H(x(T))-H(x(0))]$ is always less than the supply $\int_{0}^{T} \boldsymbol{x}^{\mathrm{T}} \boldsymbol{F} \mathrm{d} \tau$, and the dissipation which includes the equivalent resistance loss and the switching loss is $\int_{0}^{T} \boldsymbol{x}^{\mathrm{T}} \boldsymbol{R} \boldsymbol{x} \mathrm{d} \tau$. Thus, the STATCOM is strictly passive, and the controller can be designed for DT-STATCOM with the PBC theory.

\section{Passivity-based Control Design}

From equation (4), the model of STATCOM is underactuated system because the dimension of the control vectors of system $\left(u_{d}, u_{q}\right)$ is less than that of the system $\left(i_{T d}, i_{T q}, u_{d c}\right)$. Thus, DC capacitor voltage must be controlled by the indirect control method such as proportional-integral (PI) method.

By defining the desired reference current $\boldsymbol{x}^{*}=\left[\begin{array}{ll}i_{T d}{ }^{*} & i_{T q}{ }^{*}\end{array}\right]^{\mathrm{T}}$, the space vector of error system can be obtained as

$$
\boldsymbol{x}_{\boldsymbol{e}}=\boldsymbol{x}-\boldsymbol{x}^{*}=\left[\begin{array}{l}
i_{T d}{ }^{*}-i_{T d} \\
i_{T q}{ }^{*}-i_{T q}
\end{array}\right]=\left[\begin{array}{c}
i_{e T d} \\
i_{\text {eTq }}
\end{array}\right]
$$

The Lyapunov function of the error system can be described as

$$
H_{e}(x(\mathrm{~T}))=\frac{1}{2} \boldsymbol{x}_{e}^{T} \boldsymbol{M} \boldsymbol{x}_{e}
$$

Substituting (9) into (5), the dynamic error function can be described as

$$
\boldsymbol{M}\left(\dot{\boldsymbol{x}}_{e}+\dot{\boldsymbol{x}}^{*}\right)+\boldsymbol{J}\left(\boldsymbol{x}^{*}+\boldsymbol{x}_{e}\right)+\boldsymbol{R}\left(\boldsymbol{x}^{*}+\boldsymbol{x}_{e}\right)=\boldsymbol{F}
$$


Equation (11) can be rewritten as

$$
\boldsymbol{M} \dot{\boldsymbol{x}}_{e}+\boldsymbol{J} \boldsymbol{x}_{\boldsymbol{e}}+\boldsymbol{R} \boldsymbol{x}_{e}=\boldsymbol{F}-\left(\boldsymbol{M} \dot{\boldsymbol{x}}^{*}+\boldsymbol{J} \boldsymbol{x}^{*}+\boldsymbol{R} \boldsymbol{x}^{*}\right)=\boldsymbol{\xi}
$$

According to (12), the desired stable point works when $\boldsymbol{x}_{\boldsymbol{e}}=0$. The convergence speed of system is determined by the value of $\boldsymbol{R}$. It can accelerate energy dissipation of the system and make the system astringe to expected stable equilibrium point by injecting damping.

The injected dissipation damping is shown as

$$
\boldsymbol{R}_{\text {dnew }} \boldsymbol{x}_{e}=\left(\boldsymbol{R}_{d}+\boldsymbol{R}\right) \boldsymbol{x}_{e}
$$

where $\boldsymbol{R}_{\text {dnew }}$ is the expectation damping of control system, $\boldsymbol{R}_{d}$ is the injected positive definite damping. Then function (12) can be expressed as (14)

$$
\boldsymbol{M} \dot{\boldsymbol{x}}_{e}+\boldsymbol{J} \boldsymbol{x}_{\boldsymbol{e}}+\boldsymbol{R}_{\text {dnew }} \boldsymbol{x}_{e}=\boldsymbol{F}-\left(\boldsymbol{M} \dot{\boldsymbol{x}}^{*}+\boldsymbol{J} \boldsymbol{x}^{*}+\boldsymbol{R} \boldsymbol{x}^{*}-\boldsymbol{R}_{d} \boldsymbol{x}_{e}\right)=\boldsymbol{\xi}
$$

In order to decouple the d-axis and the q-axis current, let $\boldsymbol{\xi}=\boldsymbol{J} \boldsymbol{x}_{e}$. Therefore, new control law based on PBC theory can be achieved as

$$
\boldsymbol{F}-\left(\boldsymbol{M} \dot{\boldsymbol{x}}^{*}+\boldsymbol{J}\left(\boldsymbol{x}^{*}+\boldsymbol{x}_{e}\right)+\boldsymbol{R} \boldsymbol{x}^{*}-\boldsymbol{R}_{d} \boldsymbol{x}_{e}\right)=0
$$

We know that $H_{e}(x(\mathrm{~T}))>0$ because of positive definite of $\boldsymbol{M}$. The derivative of (10) is achieved as

$$
\dot{H}_{e}(x(T))=\boldsymbol{x}_{e}^{\mathrm{T}}\left(\boldsymbol{\xi}-\boldsymbol{J} \boldsymbol{x}_{e}-\boldsymbol{R}_{\text {dnew }} \boldsymbol{x}_{e}\right)=-\boldsymbol{x}_{e}^{\mathrm{T}} \boldsymbol{R}_{\text {dnew }} \boldsymbol{x}_{e}<0
$$

Dynamic error equation system (10) is stable according to Lyapunov stability theorem when $H_{e}>0, \dot{H}_{e}<0$.

Then, substitute the DT-STATCOM parameters into the new control law (17)

$\left\{\begin{array}{l}u_{d}=L \frac{d i_{T d}^{*}}{d t}-\omega L i_{T q}+R i_{T d}^{*}-R_{a 1}\left(i_{T d}-i_{T d}^{*}\right)+u_{T d} \\ u_{q}=L \frac{d i_{T q}^{*}}{d t}+\omega L i_{T d}+R i_{T q}^{*}-R_{a 2}\left(i_{T q}-i_{T q}^{*}\right)+u_{T q}\end{array}\right.$

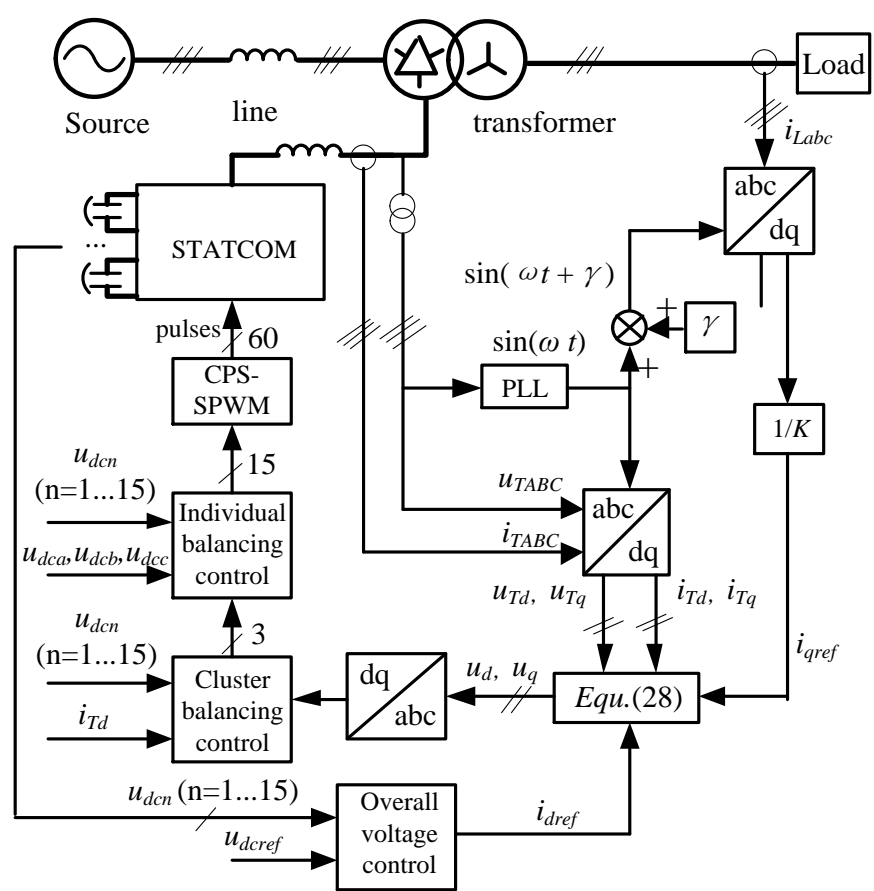

Fig.5. Diagram of the improved DT-STATCOM control system based on PBC.
Table 1 Simulation parameters

\begin{tabular}{ll}
\hline \multicolumn{1}{c}{ components } & parameters \\
\hline Grid voltage & $10[\mathrm{kV}]$ \\
Grid frequency & $50[\mathrm{~Hz}]$ \\
Line impedance & $0.5[\Omega], 1.2[\mathrm{mH}]$ \\
Transformer & $1[\mathrm{MVA}], 10 \mathrm{kV} / 0.4 \mathrm{kV}$ \\
Tap voltage & $5[\mathrm{kV}]($ central taps $)$ \\
Filter inductor & $16[\mathrm{mH}](10 \%)$ \\
Equivalent resistance of & $0.2[\Omega]$ \\
Filter inductor & \\
DC capacitor & $8000[\mu \mathrm{F}]$ \\
Cascade count per cluster & 5 \\
PWM carrier freqe cy & $2[\mathrm{kHz}]$ \\
\hline
\end{tabular}




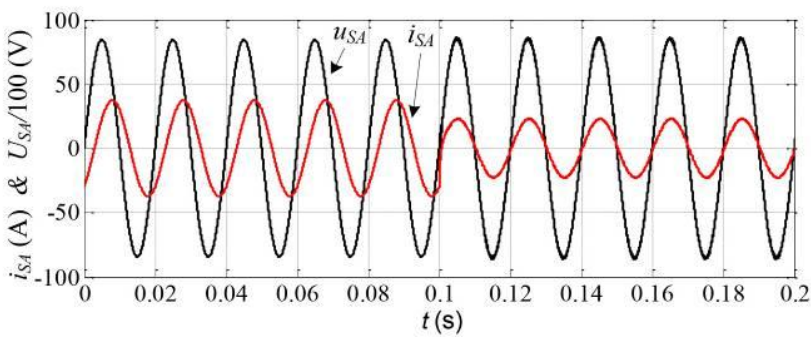

Fig. 6. Simulation results of supply voltage and current.

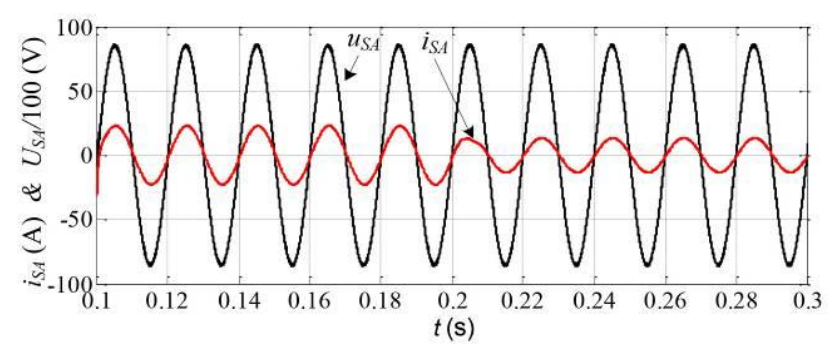

Fig. 7. Simulation results of supply voltage and current when the load decreases by $50 \%$.

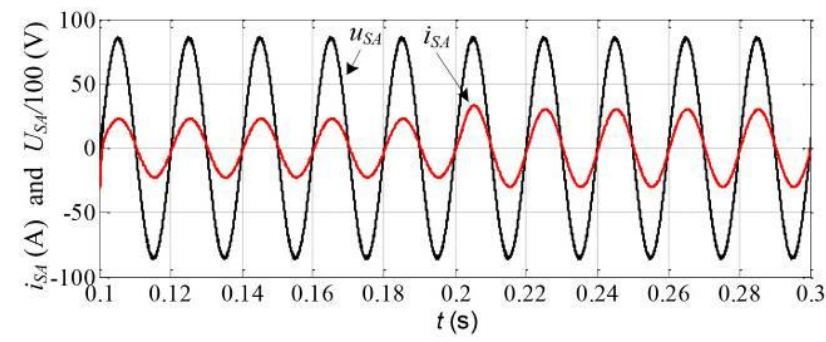

Fig. 8. Simulation results of supply voltage and current when the load increases by $50 \%$.

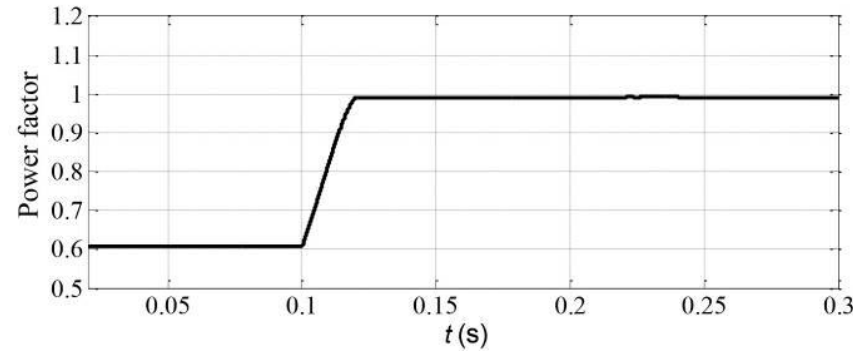

Fig. 9. The power factor of supply side

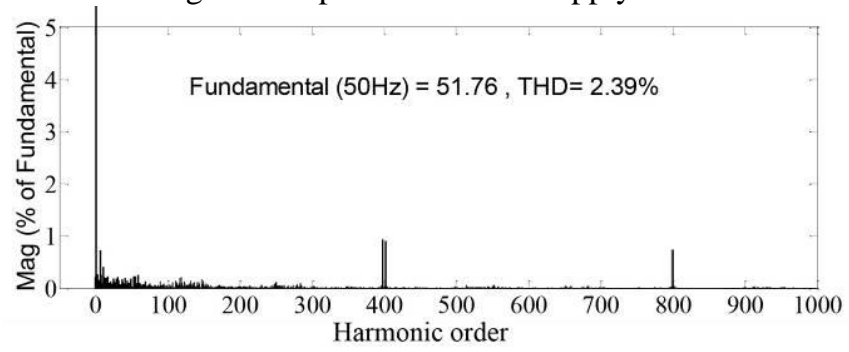

Fig. 10. Harmonic spectrum of the supply current in phase-A.

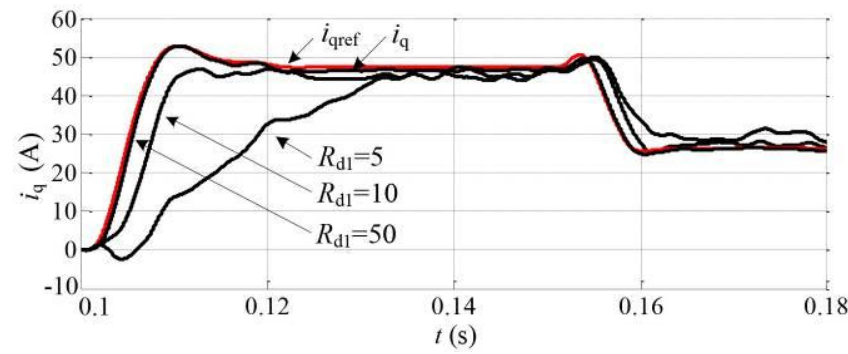

Fig. 11. Dynamic response of STATCOM with different dampings $\boldsymbol{R}_{\mathbf{d}}$

From the above analysis, Fig. 5 shows the passive controller for DT-STATCOM based on the theory of E-L to compensate the reactive power at connected taps. In addition, the cluster balancing control and individual balancing control algorithm in [10] are applied.

\section{Simulation Results}

The DT-STATCOM structure with PBC is verified by simulation in Matlab/Simulink environment. Schematic diagram of the improved DT-STATCOM system for simulation test is depicted in Fig. 5. The specific parameters of the simulation are shown in Table 1.

Fig. 6 illustrates the performance of the DT-STATCOM in response to a step load change. Specifically, it can be seen from Fig.8 that the supply voltage and current are in phase with each other after STATCOM is put into operation at $t=0.1 \mathrm{~s}$. Fig.7 shows the dynamic performance of STATCOMfor the load (inductive load) decreasing by $50 \%$ at $t=0.2 \mathrm{~s}$. Fig. 8 shows the load (inductive load) increases by $50 \%$ at $t=0.2 \mathrm{~s}$. The current of the supply side comes to steady state within $3 \mathrm{~ms}$. The dynamic response of DT-STATCOM is very fast.

Fig. 9 shows the power factor (PF) at supply side. As it can be seen, the PF increases from 0.61 to 0.99 after compensation. The harmonic spectrum of the supply current shows that total harmonic distortion (THD) is about 2.4\%, as shown in Fig. 10. Harmonic is mainly distributed in the switching frequency and its multiples spectrum.

Fig. 11 shows dynamic response of STATCOM with different injected damping $\boldsymbol{R}_{\mathbf{d}}$. It can be observed that the larger damping is chosen, the faster response of controller will get. The initial value of $R_{d 1}$ and $R_{d 2}$ are set to a relative small one and fine tune to meet the requirement of the system. The final parameters of $R_{d 1}$ and $R_{d 2}$ are $R_{d 1}=R_{d 2}=50$. 


\section{Summary}

In this paper, the mechanism and control of DT-STATCOM system are analyzed in-depth. An improved $i_{p}-i_{q}$ current detection method is proposed to eliminate the ratio and phase angle deviation caused by the transformer. The improved PBC based on E-L model is designed for inner current control loop. It can accelerate energy dissipation and improve the dynamic response of STATCOM system by injecting damping. The simulation results have verified the effectiveness of proposed DT-STATCOM. Also, the PBC method has good steady and dynamic performance. It is easy to tune the gains of the controller.

\section{Acknowledgements}

This work was financially supported by national natural science foundation of China (No. 51277084).

\section{References}

[1] Gultekin, B.; Ermis, M., "Cascaded multilevel converter-based transmission STATCOM: system design methodology and development of a $12 \mathrm{kV} \pm 12 \mathrm{MVAr}$ power stage," Power Electronics, IEEE Transactions on, vol.28, no.11, pp.4930-4950, Nov. 2013.

[2] Pereira, M., et al. "SVC PLUS: An MMC STATCOM for network and grid access applications." PowerTech, 2011 IEEE Trondheim IEEE, 2011:1-5.

[3] Bilgin, H.F.; Ermis, M., "Design and implementation of a current-source converter for use in industry applications of D-STATCOM," Power Electronics, IEEE Transactions on, vol.25, no.8, pp.1943-1957, Aug. 2010.

[4] Cetin, A.; Ermis, M., "VSC-based D-STATCOM with selective harmonic elimination," Industry Applications, IEEE Transactions on , vol.45, no.3, pp.1000-1015, May-june 2009.

[5] Wang, C.; Yin, X.; Zhang, Z.; Wen, M., "A novel compensation technology of static synchronous compensator integrated with distribution transformer," Power Delivery, IEEE Transactions on , vol.28, no.2, pp.1032-1039, Apr. 2013.

[6] Ortega R, Loria A, Nicklasson P J, et al. Passivity-based control of Euler-Lagrange systems: mechanical electrical and electromechanical application. London, UK: Springer-Verlag, 1998:15-28.

[7] Rong Xu; Yong Yu; Rongfeng Yang, et al. "A novel control method for transformerless H-bridge cascaded STATCOM with star configuration," Power Electronics, IEEE Transactions on, vol.30, no.3, pp.1189-1202, Mar. 2015.

[8] Kun Mu; Xiaoyu Ma; Xiaobin Mu; Dalei Zhu, "Study on passivity-based control of voltage source PWM DC/AC inverter," Electronic and Mechanical Engineering and Information Technology (EMEIT), 2011 International Conference on , vol.8, pp.3963-3967, Aug. 2011.

[9] Mehrasa M., Adabi M.E., Pouresmaeil E., Adabi J, "Passivity-based control technique for integration of DG resources into the power grid," Int. J. Electr. Power Energy Syst., vol 58, no.5, pp. 281-290, 2014.

[10]H. Akagi, S. Inoue and T. Yoshii, "Control and performance of a transformerless cascade PWM STATCOM with star configuration," IEEE Transactions on Industry Applications, vol. 43, no. 4, pp. 1041-1049, Jul.-Aug. 2007. 